\title{
State space method for predicting the spike times of a neuron
}

AUTHOR(S):

Kobayashi, R; Shinomoto, S

\section{CITATION:}

Kobayashi, R ...[et al]. State space method for predicting the spike times of a neuron. Physical Review E 2007, 75(1): 011925.

ISSUE DATE:

2007-01

URL:

http://hdl.handle.net/2433/49925

RIGHT:

Copyright 2007 American Physical Society 
PHYSICAL REVIEW E 75, 011925 (2007)

\title{
State space method for predicting the spike times of a neuron
}

\begin{abstract}
Ryota Kobayashi* and Shigeru Shinomoto $^{\dagger}$
Department of Physics, Kyoto University, Kyoto 606-8502, Japan

(Received 11 September 2006; revised manuscript received 26 November 2006; published 29 January 2007)

It has been established that a biological neuron reproduces the precise spike response to identical fluctuating input currents. We wish to predict the firing times of a given neuron for any input current. For this purpose, a mathematical model is introduced for mimicking the voltage response of the neuron to an input current. In predicting the firing times of a target neuron for a novel input current, we propose here the method of estimating the probability of spike occurrence, instead of naively thresholding an instantaneous value of the model voltage. The assessment is carried out maximally utilizing the information about the state space of the voltage and its time derivative(s) of the model, in advance of a possible spike, with a time lag that is determined by maximizing the mutual information. The prediction is significantly improved by the present method in comparison to the naive thresholding method.
\end{abstract}

DOI: 10.1103/PhysRevE.75.011925

PACS number(s): 87.19.La, 05.45.-a, 05.10.Gg

\section{INTRODUCTION}

The spike generation process of a neuron is a highly nonlinear transformation from the input into the output. Since Hodgkin and Huxley [1] described the ionic flux across the neuronal membrane with four nonlinear differential equations, continuous efforts have been made to construct realistic models by including more ionic channels in the model. User-friendly simulation platforms, such as NEURON [2] and GENESIS [3], enable experimental neurophysiologists to reproduce casually their experimental results or to explore potentially interesting phenomena.

In spite of such successful aspects, those realistic models have rarely been used for quantitative reproduction as well as prediction of spike times. The difficulty would be due to the complexity of the model accompanied with a large number of undetermined free parameters [4-7]. Even if a true model of a particular neuron is included in the family of models, it is difficult to find the correct set of parameters in the highdimensional space of parameters that dominate the nonlinear dynamics.

In contrast to such efforts toward the reality, there have been studies for extracting the essence of the nonlinear dynamics of neuronal spiking. A representative is the model proposed by FitzHugh [8] and Nagumo et al. [9], in which the number of equations is reduced to two: the fast and slow variables which minimally represent the excitable dynamics. The leaky integrate-and-fire model [10], originally proposed far in advance of the Hodgkin-Huxley model, consists of only one variable that corresponds to the membrane voltage, with a supplementary mechanism of voltage resetting at the firing. Those simplified models have been useful for not only extracting the essence of the dynamics, but also reducing the computational cost of studying the large-scale dynamics of an assembly of neurons, but the reality had been abandoned from the beginning.

Recently, Kistler et al. [11,12] suggested extending the leaky integrate-and-fire model so that real dynamics of any

\footnotetext{
*Electronic address: kobayashi@ton.scphys.kyoto-u.ac.jp

†Electronic address: shinomoto@scphys.kyoto-u.ac.jp
}

neuron are adopted. The so-called "spike response model" has been successful in not only reproducing the data but also in predicting the spike times for a novel input current [11-15]. Due to the linearity of the model, the integration kernel can be learned easily from sample data. The fact that such a simple model could achieve a fairly precise prediction indicates that the spike occurrence is determined principally by the simple subthreshold dynamics. In other words, the highly nonlinear dynamics of a neuron can be decomposed into two predictable processes: a relatively simple subthreshold dynamics and an action potential of a nearly fixed shape (Fig. 1).

We propose in the present paper a framework of improving the prediction of spike times by paying close attention to the transfer between the two predictable processes. It is assumed in the original spike response model that a spike occurs if the voltage exceeds a certain threshold [12]. We revised this rule so as to utilize the information about not only the instantaneous voltage, but also its time derivative(s). Such a subthreshold state can provide cues for the occurrence of a spike, with a certain time difference. The time difference is chosen so that the mutual information between the subthreshold state and the occurrence of a spike is maximized.

By adopting a simple linear filter model (LFM) [16] and the spike response model (SRM) [12] for mimicking the sub-

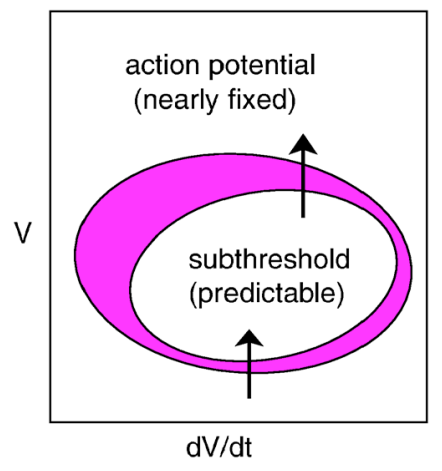

FIG. 1. (Color online) The highly nonlinear dynamics of a neuron is decomposed into two simple, predictable processes. 
threshold voltage response of a neuron, we examine how much the present framework may improve the prediction for simulation data of a fast-spiking neuron model [17] and an intrinsic bursting neuron model [18].

\section{STATE SPACE METHOD}

\section{A. Deterministic feature of the spike generation}

Biological neurons precisely reproduce the spike response when presented with identical fluctuating input currents [19]. This implies that the neuronal membrane potential $V(t)$ is uniquely determined by the past input current $I(t)$, or

$$
V(t)=F[I(t)],
$$

where $F[I(t)]$ represents a functional of a time-dependent current $I(t)$. The spike; the rapid swing in the polarity of the membrane potential, could be defined practically by measuring the membrane potential $V(t)$ exceeding a certain threshold,

$$
V(t)>V_{\text {th }} .
$$

The time of each spike could be defined empirically either as the first time the threshold is exceeded or as the peak of the action potential that follows the crossing. We adopted the latter definition in the present study.

We will denote in the following the output of a model (in the lower case) as

$$
v_{k}(t)=f_{k}[I(t)]
$$

where $k$ represents a set of model parameters. The model parameters are learned by mimicking sample input-output data. This is achieved by minimizing the integrated square error,

$$
E_{k}=\int\left[V(t)-v_{k}(t)\right]^{2} d t
$$

\section{B. The state space}

No simplified threshold model is able to perfectly reproduce the output $V(t)$ of a target neuron for a given input $I(t)$, even in the learning stage. As the output of the model $v_{k}(t)$ is not identical to the true membrane potential of the target neuron $V(t)$, a spike occurrence cannot be obtained by simply applying the same threshold rule Eq. (2) to $v_{k}(t)$. In this paper, we suggest revising the prediction method so that a spike occurrence is best predicted by observing the dynamics of the model potential $v_{k}(t)$.

Suppose that we have adjusted the parameters of the model so that its output $\left\{v_{k}(t)\right\}$ best approximates the membrane potential of a target neuron $\{V(t)\}$ for a given set of currents $\{I(t)\}$. If the sample data set $\{I(t), V(t)\}$ employed in learning is large enough, the spike occurrence can be predicted by estimating an empirical probability of a spike being generated at the time $t$, given a time-dependent orbit of an estimated output, $\left\{v_{k}(t)\right\}$, as

$$
p_{\text {spike }}\left[t \mid\left\{v_{k}(t)\right\}\right] .
$$
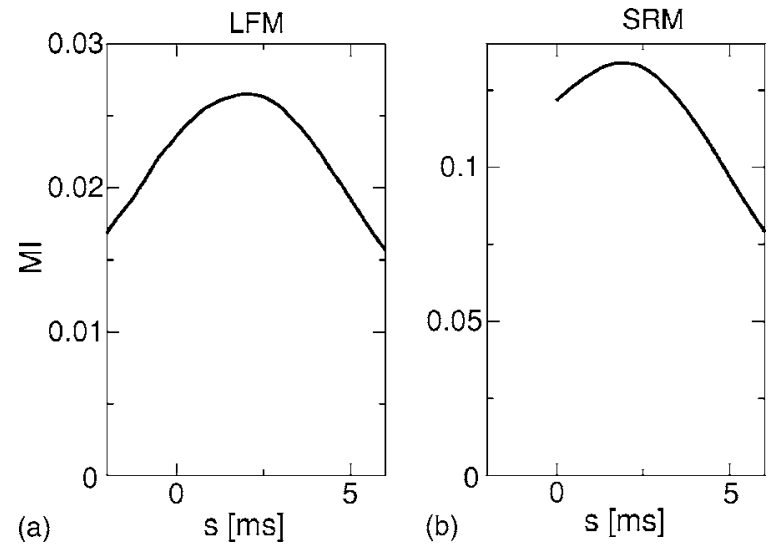

FIG. 2. The mutual information $\mathcal{I}\left(z_{t} ; \vec{v}_{t-s}\right)$ between the estimated potential and the occurrence of a spike (computed for the fast spiking neuron model with the current I). (a) linear filter model (LFM) and (b) spike response model (SRM).

In a practical experiment, however, the amount of collectable data is insufficient for estimating the spiking probability with respect to any orbit of $v_{k}(t)$. In place of such exhaustive examination, we suggest utilizing the state space information such as the time derivatives of the model potential at a certain time. The spike occurrence at time $t$ could be predicted from the $m$-dimensional state space information $\vec{v}$ $\equiv\left(v, v^{\prime}, \ldots, v^{(m-1)}\right)$, as observed at a time $s$ before $t$, as

$$
p_{\text {spike }}\left(t \mid \vec{v}_{t-s}\right) \text {, }
$$

where $\vec{v}_{t-s} \equiv\left[v(t-s), v^{\prime}(t-s), \ldots, v^{(m-1)}(t-s)\right]$.

\section{The determination of the time shift}

The time shift $s$ introduced in the spike time prediction, Eq. (6), is chosen to make the prediction more reliable. We propose optimizing the time shift $s$ by maximizing the mutual information between the state space information $\vec{v}_{t-s}$ and the presence or absence of a spike at a time interval $(t$ $-\delta t / 2, t+\delta t / 2]$, which is denoted as $z_{t}=1$ or 0 . The mutual information [20] is given as

$$
\mathcal{I}\left(z_{t} ; \vec{v}_{t-s}\right)=\mathcal{I}\left(\vec{v}_{t-s} ; z_{t}\right)=H\left(\vec{v}_{t-s}\right)-H\left(\vec{v}_{t-s} \mid z_{t}\right),
$$

where

$$
\begin{gathered}
H\left(\vec{v}_{t-s}\right)=-\int d \vec{v}_{t-s} p\left(\vec{v}_{t-s}\right) \log p\left(\vec{v}_{t-s}\right), \\
H\left(\vec{v}_{t-s} \mid z_{t}\right)=-\sum_{z_{t} \in\{0,1\}} \int d \vec{v}_{t-s} p\left(\vec{v}_{t-s} \mid z_{t}\right) p\left(z_{t}\right) \log p\left(\vec{v}_{t-s} \mid z_{t}\right) .
\end{gathered}
$$

Here, $p\left(\vec{v}_{t-s} \mid z_{t}\right)$ is the probability, given the presence or absence of a spike at time $t \in(t-\delta t / 2, t+\delta t / 2]$, of the state being $\vec{v}_{t-s}$, a time $s$ before the spike.

The mutual information $\mathcal{I}\left(z_{t} ; \vec{v}_{t-s}\right)$ computed with sample data according to Eq. (7) is depicted in Fig. 2, whose maximum position determines the optimal time shift $s$. With the 
time difference $s$ optimized, we then obtain the empirical probability of the spike occurrence at the time $t$, given the state space information at the time $t-s$, using the Bayes theorem,

$$
p_{\text {spike }}\left(t \mid \vec{v}_{t-s}\right) \propto p\left(z_{t}=1 \mid \vec{v}_{t-s}\right)=\frac{p\left(\vec{v}_{t-s} \mid z_{t}\right) p\left(z_{t}\right)}{p\left(\vec{v}_{t-s}\right)},
$$

with which we predicted the spike occurrence.

\section{APPLICATION OF THE STATE SPACE METHOD}

\section{A. Target neurons}

We evaluated our state space method of predicting spike times by applying it to target data obtained for two neuron models: a fast-spiking neuron model proposed by Erisir $\mathrm{et} \mathrm{al}$. [17] which is adapted by Jolivet et al. [13] and an intrinsic bursting neuron model proposed by Izhikevich [18].

In those virtual experiments, two independent fluctuating currents characterized by the same mean and standard deviation are injected to each model neuron to obtain two sets of input-output data $\{I(t), V(t)\}$. A prediction model was first trained using one sample data set. Then, the model parametrized with the first sample data is used to predict the output of the second input current that is independent of the first.

Predictive performance was evaluated with the benchmark of Kistler et al. [11], the "coincidence factor,"

$$
\Gamma(\Delta)=\frac{N_{\text {coinc }}-\left\langle N_{\text {coinc }}\right\rangle}{\frac{1}{2}\left(N_{\text {data }}+N_{\text {model }}\right)} \frac{1}{1-2 \nu \Delta},
$$

where $N_{\text {data }}$ and $N_{\text {model }}$, respectively, represent the numbers of spikes in the original data and prediction model, $N_{\text {coinc }}$ is the number of coincident spikes with the precision of $\Delta$, and $\left\langle N_{\text {coinc }}\right\rangle=2 \nu \Delta N_{\text {data }}$ is the expected number of coincidences of the data and the Poisson spikes of the same rate $\nu . \Delta$ is chosen as 2 [ms] in accordance with Jolivet et al. [13].

\section{B. Prediction models}

In the present study, we adopted a linear filter model [16] and the spike response model [12] as prediction models. The linear filter model, which we refer to as LFM here, simply filters the input current in the manner

$$
f_{k}[I(t)]=\int_{0}^{\infty} K\left(t^{\prime}\right) I\left(t-t^{\prime}\right) d t^{\prime}+v_{0},
$$

whose parameters consist of the shape of the kernel $K(t)$ and the constant $v_{0}$. This LFM does not assume resetting the potential.

Contrastingly, the spike response model, which is referred to as SRM, resets the potential on some presumed condition of firing and restarts integrating the input current in the manner

$$
f_{k}[I(t)]=\int_{0}^{t-t_{f}} K\left(t^{\prime}\right) I\left(t-t^{\prime}\right) d t^{\prime}+\sum_{j=1}^{f} H\left(t-t_{j}\right),
$$

where $\left\{t_{1}, t_{2}, \ldots, t_{f}\right\}$ represent the times of (presumed) firings. The parameters of the SRM include the shapes of the kernel
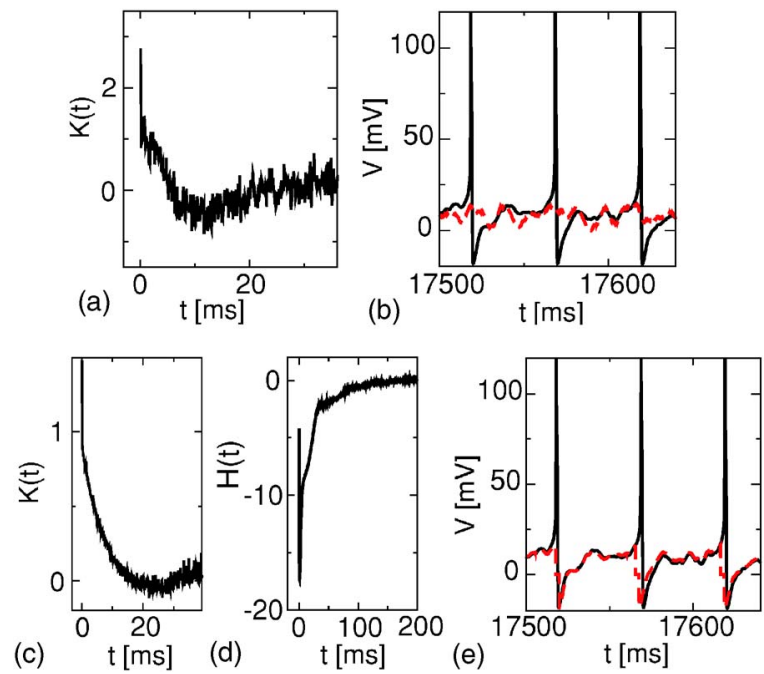

FIG. 3. (Color online) (a) The kernel $K(t)$ estimated with LFM. (b) The estimated voltage $v_{k}(t)$ (dashed line), in reference to the target voltage $V(t)$ produced by the fast-spiking neuron model (solid line). (c)-(e) The kernel $K(t)$, postspike hyperpolarizing potential $H(t)$, and the voltage $v_{k}(t)$ estimated with SRM.

$K(t)$ and a postspike hyperpolarizing potential $H(t)$, and a threshold in determining the firing (resetting) time.

The parameters of these models are adjusted while mimicking the input and output so that the integrated square error, Eq. (4), is minimized in the nonparametrical fashion. Figures 3(a) and 3(b) depict $K(t)$ and $v_{k}(t)$ of the LFM; (C)-(E) depict $K(t), H(t)$, and $v_{k}(t)$ of the SRM.

\section{Predicting the spike times}

Based on the estimated voltage $v_{k}(t)$ with respect to sample data, we compute the empirical probabilities, $p\left(\vec{v}_{t-s}\right)$, $p\left(\vec{v}_{t-s} \mid z_{t}\right)$, and $p\left(z_{t}\right)$ for two-dimensional state space information $\vec{v}_{t-s} \equiv\left[v(t-s), v^{\prime}(t-s)\right]$.

In computing empirical probabilities, we quantized the two-dimensional phase space $\vec{v} \equiv\left(v, v^{\prime}\right)$ and the time. In the discretized time, we counted the occurrence of a spike, $z_{t}$ $=1$, for the bins in which the true membrane potential $V(t)$ exceeds a threshold $V_{t h}$. We confirmed that the results were robust against the choice of the threshold over a fairly wide range, and fixed the threshold as $V_{\mathrm{th}}=20[\mathrm{mV}]$.

With a sufficiently small time step (we adopted $\delta t$ $=0.1[\mathrm{~ms}]$ ), a single spike is transformed into a succession of spike occurrences $z_{t}=1$. In the case of the fast spiking neuron, the number of $z_{t}=1$ in each spike was about 20 (2[ms]) and $40(4[\mathrm{~ms}])$ for the current I and current II, whose characteristics will be described later. The time shift $s$ is determined so that the mutual information $\mathcal{I}\left(z_{t} ; \vec{v}_{t-s}\right)$ given by Eq. (7) is maximized with respect to training data.

In computing the empirical spiking probability, we further smoothed out the raw $p\left(z_{t} \mid \vec{v}_{t-s}\right)$ with the Gaussian kernel in time with the variance of $\sigma_{p}^{2}$ (we adopted $\sigma_{p}=4[\mathrm{~ms}]$ for the LFM and 1 [ms] for the SRM) as 
TABLE I. The values of maximum mutual information and the optimal time shift computed for the fast spiking neuron model with two types of currents I and II.

\begin{tabular}{lccc}
\hline \hline Current & Model & $\mathcal{I}_{\max }$ & $s[\mathrm{~ms}]$ \\
\hline Current I & LFM & $2.7 \times 10^{-2}$ & 2.0 \\
Current I & SRM & $1.3 \times 10^{-1}$ & 1.8 \\
Current II & LFM & $5.4 \times 10^{-2}$ & 1.2 \\
Current II & SRM & $1.0 \times 10^{-1}$ & 0.9 \\
\hline \hline
\end{tabular}

$$
p_{\text {spike }}(t) \equiv \sum_{j} \frac{1}{\sqrt{2 \pi \sigma_{p}^{2}}} \exp \left(-\frac{(j \delta t)^{2}}{2 \sigma_{p}^{2}}\right) p\left(z_{t+j}=1 \mid \vec{v}_{t+j-s}\right) \delta t
$$

and presumed that a spike would be present when $p_{\text {spike }}(t)$ is greater than a certain threshold $p_{\text {spike. Though a theoretical }}^{*}$ method for searching the threshold parameter is suggested [21], in this paper we employed the empirical method; i.e., the threshold parameter was searched for to maximize the coincidence factor $\Gamma$.

\section{THE PERFORMANCE OF THE PREDICTION METHOD}

In this section, we show the performance of our statespace method by applying it to target data produced by the fast spiking neuron model $[13,17]$ and the intrinsic bursting neuron model [18]. The details of the neuron models are described in the Appendixes.

\section{A. Fast-spiking neuron model}

For the fast-spiking neuron model, we examined two kinds of fluctuating currents characterized with different means and standard deviations generated by the OrnsteinUhlenbeck process: (current I) the mean $\mu=3.0[\mu \mathrm{A}]$, the standard deviation $\sigma=1.0[\mu \mathrm{A}]$, and the time scale of the fluctuation $\tau=2$ [ms]; (current II) the mean $\mu=1.0[\mu \mathrm{A}]$, the standard deviation $\sigma=3.0[\mu \mathrm{A}]$, and the time scale of the fluctuation $\tau=2[\mathrm{~ms}]$. For each current statistic, either I or II, we generated two independent realizations of currents; one for the training and the other for testing the generalization ability.

Table I summarizes the values of maximum mutual information computed according to Eq. (7) and the optimal time shift computed for the two types of currents I and II, using the LFM and the SRM. For each current, the optimal time shifts determined with two models were similar, but the mutual information is larger for SRM.

Figure 4 compares the results of spike time prediction achieved by a naive thresholding method and our state space method, in reference to the original spike times, respectively, using the LFM and the SRM. It is observed from this figure that the prediction based on the state space method is more accurate and robust than that of the thresholding method. Figure 5 depicts a state space orbit of a target neuron for an instance of the spike generation, and compares orbits of the

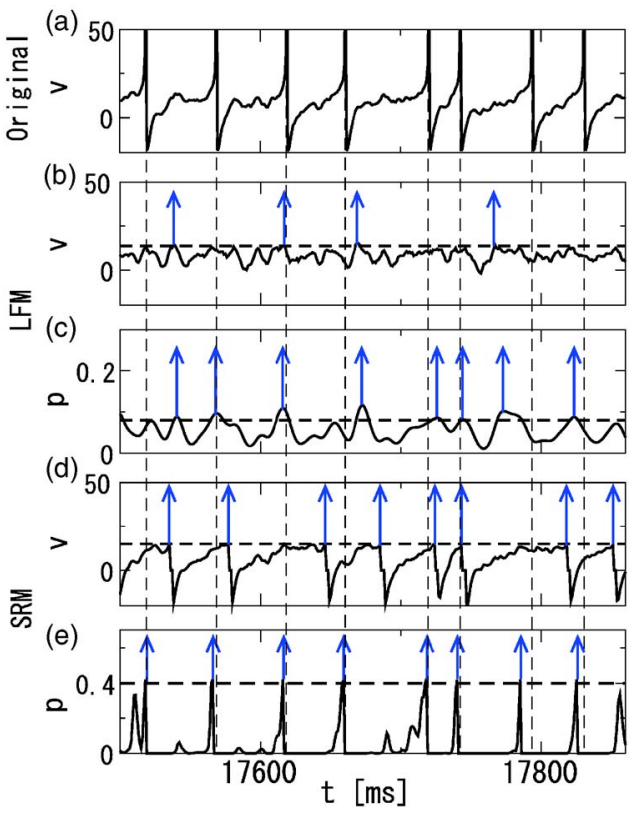

FIG. 4. (Color online) Comparison of the spike time predictions obtained with the LFM and the SRM for the fast-spiking neuron. (a) The target membrane potential $V(t)$. (b) and (d) Spikes predicted (vertical arrows) by naively thresholding the model potential of the LFM and the SRM. (c) and (e) Spikes predicted by the present state space method.

LFM and the SRM that try to mimic it. The prediction model can mimic the target orbit in the subthreshold region but fails to catch the spiking orbit in the suprathreshold region. The spike occurrence is predicted by estimating the conditional probability, Eq. (10), given the state $\left(v, v^{\prime}\right)$ of the prediction model. In the present framework, the state space information about the probability of spiking is effectively used for pre- (b)

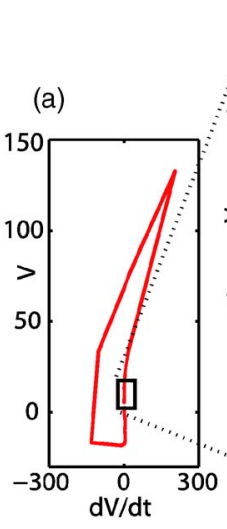

(c)

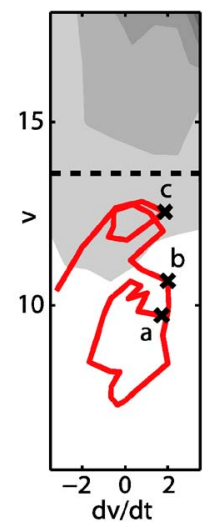

(d)

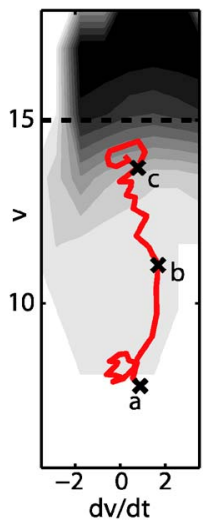

FIG. 5. (Color online) (a) An orbit of a target fast spiking neuron for an instance of the spike generation in the state space of $\left(V, V^{\prime}\right)$ (from 17810 to 17840 [ms] of Fig. 4). (b) Magnified view. (c) and (d) The orbits of the prediction models LFM and SRM that try to mimic the target orbit. Contours represent the probability of spike occurrence computed with the Bayes formula, Eq. (10). Three points $a, b$, and $c$ in the spaces of $\left(V, V^{\prime}\right)$ and $\left(v, v^{\prime}\right)$ represent the states of identical times, respectively, $t=17810,17818$, and 17822 [ms]. 
TABLE II. The coincidence factors evaluated for the fastspiking neuron with various methods of prediction; naive thresholding method, the state space method without a time shift, and the state space method with the time shift optimized.

\begin{tabular}{lccc}
\hline \hline Model & Method & Current I & Current II \\
\hline LFM & Naive thresholding & 0.15 & 0.49 \\
LFM & State space $(s=0)$ & 0.27 & 0.58 \\
LFM & State space $(s$ : optimized) & 0.30 & 0.59 \\
SRM & Naive thresholding & 0.42 & 0.74 \\
SRM & State space $(s=0)$ & 0.48 & 0.75 \\
SRM & State space $(s:$ optimized $)$ & 0.57 & 0.77 \\
\hline \hline
\end{tabular}

dicting spike times. The contour lines for higher probabilities of spiking for the LFM resemble an ad hoc "dynamic spike threshold" introduced by Azouz and Gray [22], in which $v$ drops with $d v / d t$ along the contour lines. However, it is noteworthy that the contour lines for lower probabilities for the LFM, as well as all the contour lines for the SRM are inversely curved: $v$ increases with $d v / d t$ along the contour lines.

The coincidence factors $\Gamma$ evaluated for a naive thresholding method and the state space method based on the LFM and the SRM are summarized in Table II. The prediction is significantly improved by our method, due to not only examining the state space but also introducing the time shift.

\section{B. Intrinsic bursting neuron model}

For the intrinsic bursting neuron model, we also examined two kinds of fluctuating currents: (current III) the mean $\mu$ $=7.5[\mu \mathrm{A}]$, the standard deviation $\sigma=2.5[\mu \mathrm{A}]$, and the time scale of the fluctuation $\tau=2[\mathrm{~ms}]$; (current IV) the mean $\mu$ $=2.5[\mu \mathrm{A}]$, the standard deviation $\sigma=7.5[\mu \mathrm{A}]$, and the time scale of the fluctuation $\tau=2[\mathrm{~ms}]$. Due to the significant difference between the model neurons, we had to adopt here the currents different from those applied to the fast spiking neurons. For each current statistic, either III or IV, we also generated two independent realizations of currents; one for the training and the other for testing the generalization ability.

Table III summarizes the values of maximum mutual information and the optimal time shift computed for the two types of currents III and IV, using the LFM and the SRM. Interestingly, the optimal time shift $s$ is crucially dependent on the prediction model (LFM, SRM) in this case.

TABLE III. The values of maximum mutual information and the optimal time shift computed for the intrinsic bursting neuron with two types of currents III and IV.

\begin{tabular}{lccc}
\hline \hline Current & Model & $\mathcal{I}_{\max }$ & $s[\mathrm{~ms}]$ \\
\hline Current III & LFM & $3.1 \times 10^{-2}$ & 0.6 \\
Current III & SRM & $6.0 \times 10^{-2}$ & 2.2 \\
Current IV & LFM & $5.8 \times 10^{-2}$ & 0.7 \\
Current IV & SRM & $7.2 \times 10^{-2}$ & 1.2 \\
\hline \hline
\end{tabular}

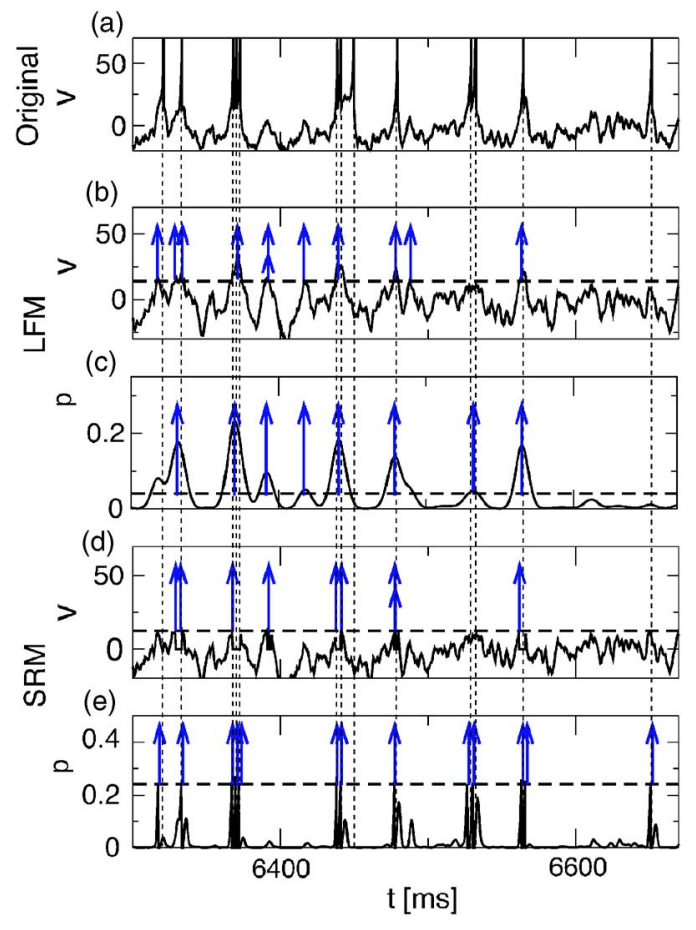

FIG. 6. (Color online) Comparison of the spike time predictions obtained with the LFM and the SRM for the intrinsic bursting neuron. (a)-(e) are the same as in Fig. 4.

Figure 6 compares the results of spike time prediction achieved by a naive thresholding method and our state space method. As in the case of the fast-spiking neuron, the prediction based on the state space method is more accurate and robust than that of the naive thresholding method. It is noteworthy that the detailed feature of the bursting was precisely predicted by applying our state space method to SRM. Figure 7 depicts a state space orbit of a target neuron for an instance of the spike generation and compares orbits of the LFM and the SRM that try to mimic it. In the case of the SRM, the contour lines for the probability of spiking is significantly curved. This indicates the possibility that the pre-

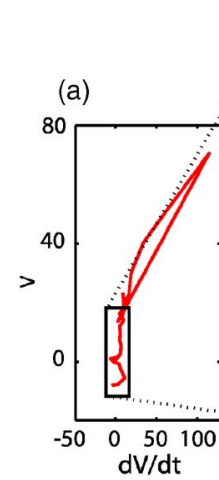

(b)

(c)

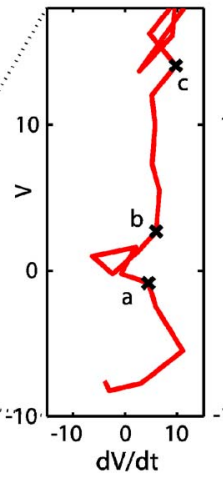

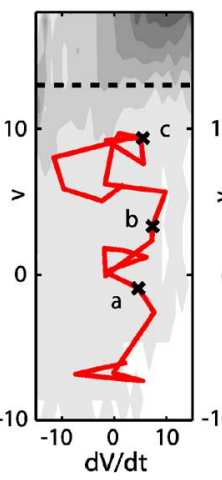

(d)

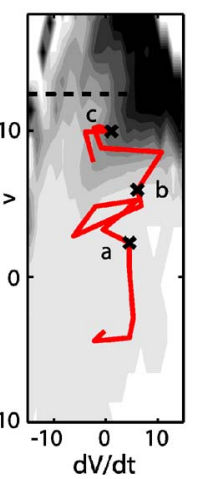

FIG. 7. (Color online) (a) An orbit of a target intrinsic bursting neuron for an instance of the spike generation in the state space of $\left(V, V^{\prime}\right)$ (from 6520 to 6530 [ms] of Fig. 6). (b)-(d) are the same as in Fig. 5. Three points $a, b$, and $c$ represent the states of identical times, respectively, $t=6522,6525$, and 6527 [ms]. 
TABLE IV. The coincidence factors evaluated for the intrinsic bursting neuron.

\begin{tabular}{lccc}
\hline \hline Model & Method & Current III & Current IV \\
\hline LFM & Naive thresholding & 0.32 & 0.52 \\
LFM & State space $(s=0)$ & 0.40 & 0.55 \\
LFM & State space $(s$ : optimized $)$ & 0.40 & 0.56 \\
SRM & Naive thresholding & 0.26 & 0.56 \\
SRM & State space $(s=0)$ & 0.27 & 0.59 \\
SRM & State space $(s:$ optimized $)$ & 0.44 & 0.76 \\
\hline \hline
\end{tabular}

diction is improved significantly with our state space method in which the information of $d v / d t$ is utilized. The improvement is actually more significant in the SRM case than in the LFM case, as seen in Table IV.

\section{DISCUSSION}

We proposed a method of evaluating the probability of the spike occurrence by observing the state space of the voltage and its time derivative(s) in advance of the possible spike time. The prediction was significantly improved by the state space method compared to the prediction obtained by naively thresholding an instantaneous value of the estimated potential.

The state space examined in this paper was twodimensional, $\vec{v}=\left(v, v^{\prime}\right)$. Possible extension to the higher dimensional space constitutes an interesting issue, especially, the exploration of the optimal spatial dimension for prediction, for a given set of data. The application of the present method to the real data is the most interesting problem. We recently started to work in this direction and categorize neurons based on their spiking mechanisms.

The state space method developed here is a rather general framework that may be applicable to any nonlinear phenomena composed of locally predictable dynamics, and there are studies that bear some similarity to ours. In the following, we would like to discuss the relationships of the existing methods to the present method.

\section{A. The escape rate model}

Biological neurons reproduce the spike response for identical fluctuating input currents fairly precisely, but there still remains a certain variability. The "escape rate model" $[12,23]$ tries to represent such variability by replacing the deterministic thresholding principle with the following stochastic principle: By assuming the "voltage dependent instantaneous probability of firing" to be of the form of

$$
f\left(v(t)-v_{\mathrm{th}}\right)=\exp \left(\frac{v(t)-v_{\mathrm{th}}}{\delta v}\right),
$$

the firing probability is explicitly obtained as

$$
p_{\text {spike }}(t)=1-\exp \left[-\delta t f\left(v(t)-v_{\text {th }}\right)\right],
$$

where $\delta t$ is a time bin. Jolivet et al. estimated this probability from experimental data [15].

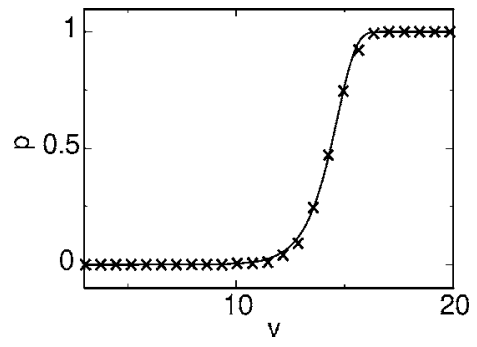

FIG. 8. Crosses: the firing probability $p\left(z_{t}=1 \mid v_{t-s}\right)$ for predicting the fast-spiking neuron with the SRM computed using the Bayes theorem Eq. (10) $(\delta t=0.1[\mathrm{~ms}])$. Solid line: the escape rate model Eq. (16) fitted to the Bayes probability $\left(\delta v=1.15, v_{\text {th }}=12.7\right)$.

Our state space method can be viewed as giving the deeper foundation of this stochastic principle, by deriving the firing probability from the Bayes theorem, as Eq. (10). The firing rule similar to Eq. (16) can be obtained with our state space method, by reducing the state space $\vec{v}$ $\equiv\left(v, v^{\prime}, \ldots, v^{(m-1)}\right)$ to be one-dimensional, $m=1$, and ignoring the time shift, $s=0$. Figure 8 compares the firing probabilities of our state space method and the escape rate model, estimated for the fast-spiking neuron using the SRM. It is observed that the above escape rate model (solid line) can be fitted to the probability computed by our method (crosses).

\section{B. The generalized spike triggered average}

Agüera y Arcas et al. [24] proposed a method for estimating kernel(s) for predicting spike times, based on the linearnonlinear-Poisson (LNP) framework [25,26]. They assumed that the firing probability can be represented as

$$
\begin{gathered}
p_{\text {spike }}(t)=g\left(x_{1}, x_{2}, \ldots, x_{D}\right), \\
x_{i}=\int f_{i}(s) I(t-s) d s(i=1,2, \ldots, D),
\end{gathered}
$$

and proposed the method to determine $f_{i}(s)$ and $g\left(\left\{x_{i}\right\}\right)$ from the input current $I(t)$ and the observed spike times $t_{j}(j$ $=1,2, \ldots, n)$.

Our method applied to the LFM can be mapped to their framework by regarding as $f_{m}(t)=K^{(m)}(t)$ in Eq. (18) and

$$
v^{(m)}(t)=\int K^{(m)}(s) I(t-s) d s(m=0,1,2, \ldots) .
$$

In our framework, the kernel was directly determined from the input-output data $\{I(t), V(t)\}$. It would be interesting to compare our kernel with the kernel estimated from $I(t)$ and spike times according to the method of Aguiera y Arcas et al. [24].

Our method applied to the SRM can be rewritten as

$$
\begin{gathered}
v^{(m)}(t)=\int K^{(m)}(s) I(t-s) d s+\sum_{j} H^{(m)}\left(t-t_{j}\right) \\
(m=0,1,2, \ldots) .
\end{gathered}
$$

In this case, the effects of the past spikes are taken into account by the term of $H^{(m)}\left(t-t_{f}\right)$, and the model is beyond 
TABLE V. The parameters for the fast-spiking model. The membrane capacity is $C=1.0\left[\mu \mathrm{F} / \mathrm{cm}^{2}\right]$.

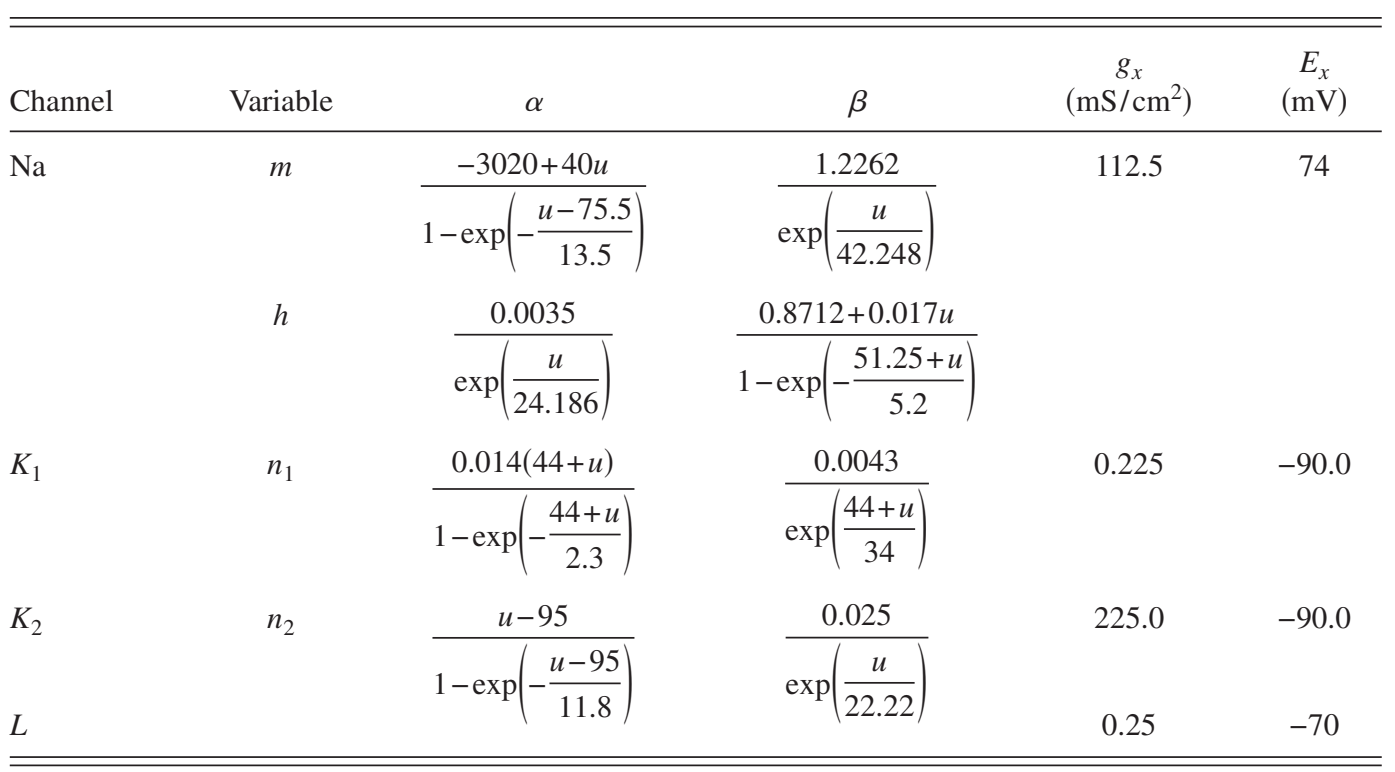

the simple projection operation of their suggestion. It would be nevertheless interesting to extend the present analysis to a higher dimensional state space, or to a curved space, as was suggested by them.

\section{ACKNOWLEDGMENTS}

This study was supported in part by Grants-in-Aid for Scientific Research to S.S. from the Ministry of Education, Culture, Sports, Science and Technology of Japan (16300068 and 18020015) and the 21st Century COE "Center for Diversity and Universality in Physics."

\section{APPENDIX A: FAST-SPIKING NEURON MODEL}

The fast-spiking neuron model proposed by Erisir et al. [17] was used in this contribution as a (virtual) target experiment. The details of the model were adapted here to Jolivet et al. [13] to allow the direct comparison of the performances. Specifically, the model is described as

$$
\begin{gathered}
C \frac{d u(t)}{d t}=-\left[I_{\mathrm{Na}}+I_{\mathrm{K}_{1}}+I_{\mathrm{K}_{2}}+I_{\mathrm{L}}\right]+I^{\mathrm{ext}}(t), \\
I_{\mathrm{Na}}=g_{\mathrm{Na}} m^{3} h\left(u-E_{\mathrm{Na}}\right), \\
I_{\mathrm{K}_{1}}=g_{\mathrm{K}_{1}} n_{1}^{4}\left(u-E_{\mathrm{K}}\right), \quad I_{\mathrm{K}_{2}}=g_{\mathrm{K}_{2}} n_{2}^{2}\left(u-E_{\mathrm{K}}\right),
\end{gathered}
$$

$$
I_{\mathrm{L}}=g_{\mathrm{L}}\left(u-E_{\mathrm{L}}\right),
$$

where the gate variables $x \equiv n_{1}, n_{2}, m$, and $h$ obey the differential equations of the form

$$
\frac{d x}{d t}=\alpha_{x}(u)(1-x)-\beta_{x}(u) x,
$$

whose parameters $\alpha_{x}(u)$ and $\beta_{x}(u)$ are functions of $u$, as listed in Table V.

\section{APPENDIX B: INTRINSIC BURSTING NEURON MODEL}

Another target neuron we adopted is the Izhikevich model that exhibits intrinsic bursting [18]. Specifically, the model is described as

$$
\begin{gathered}
\frac{d u(t)}{d t}=0.04 u^{2}+5 u+140-w+I, \\
\frac{d w(t)}{d t}=a(b u-w),
\end{gathered}
$$

where $u$ and $w$ represent the membrane potential and a membrane recovery variable, respectively, with auxiliary afterspike resetting mechanisms,

$$
\text { if } v \geqslant 30[\mathrm{mV}], \quad \text { then }\left\{\begin{array}{l}
u \rightarrow c, \\
w \rightarrow w+d .
\end{array}\right.
$$

The parameters were chosen as $a=0.02, b=0.2, c=-55$, and $d=4$.
[1] A. L. Hodgkin and A. F. Huxley, J. Physiol. (London) 117, 500 (1952)

[2] M. L. Hines and N. T. Carnevale, Neural Comput. 9, 1179 (1997).
[3] J. M. Bower and D. Beeman, The Book of GENESIS: Exploring Realistic Neural Models with the GEneral NEural SImulation System (Springer-Verlag, New York, 1995).

[4] Y. Tsubo, T. Kaneko, and S. Shinomoto, Neural Networks 17, 
165 (2004).

[5] Q. J. M. Huys, M. B. Ahrens, and L. Paninski, J. Neurophysiol. 96, 872 (2006).

[6] P. Achard and E. De Schutter, PLoS Comput. Biol. 2, e94 (2006).

[7] A. A. Prinz, C. P. Billimoria, and E. Marder, J. Neurophysiol. 90, 3998 (2003).

[8] R. FitzHugh, Biophys. J. 1, 445 (1961).

[9] J. Nagumo, S. Arimoto, and S. Yoshizawa, Proc. IRE 50, 2061 (1962)

[10] L. Lapicque, J. Physiol. Pathol. Gen. 9, 620 (1907).

[11] W. Kistler, W. Gerstner, and J. L. van Hemmen, Neural Comput. 9, 1015 (1997).

[12] W. Gerstner and W. Kistler, Spiking Neuron Models (Cambridge Univ. Press, Cambridge, England, 2002).

[13] R. Jolivet, T. J. Lewis, and W. Gerstner, J. Neurophysiol. 92, 959 (2004).

[14] R. Jolivet, A. Rauch, H. R. Lüscher, and W. Gerstner, in Advances in Neural Information Processing Systems 18, edited by Y. Weiss, B. Schölkopf, and J. Platt (MIT Press, Cambridge, MA, 2006), pp. 595-602.

[15] R. Jolivet, A. Rauch, H. R. Lüscher, and W. Gerstner, J. Com- put. Neurosci. 21, 35 (2006).

[16] D. T. Westwick and R. E. Kearney, Identification of Nonlinear Physiological Systems (IEEE Press Series in Biomedical Engineering) (Wiley-IEEE Press, Piscataway, 2003).

[17] A. Erisir, D. Lau, B. Rudy, and C. S. Leonard, J. Neurophysiol. 82, 2476 (1999).

[18] E. M. Izhikevich, IEEE Trans. Neural Netw. 14, 1569 (2003).

[19] Z. F. Mainen and T. J. Sejnowski, Science 268, 1503 (1995).

[20] D. MacKay, Information Theory, Inference and Learning Algorithms (Cambridge Univ. Press, Cambridge, England, 2003).

[21] L. Paninski, J. W. Pillow, and E. P. Simoncelli, Neurocomputing 65-66, 379 (2005).

[22] R. Azouz and C. M. Gray, Proc. Natl. Acad. Sci. U.S.A. 97, 8110 (2000).

[23] H. E. Plesser and W. Gerstner, Neural Comput. 12, 367 (2000).

[24] B. Agüera y Arcas, A. L. Fairhall, and W. Bialek, Neural Comput. 15, 1715 (2003).

[25] E. J. Chichilnisky, Network Comput. Neural Syst. 12, 199 (2001).

[26] J. W. Pillow, L. Paninski, V. J. Uzzell, E. P. Simoncelli, and E. J. Chichilnisky, J. Neurosci. 25, 11003 (2005). 